\title{
Rouille argentée des bananes martiniquaises : distribution et méthode de lutte
}

Frédéric Rey

15 rue Pasteur, 11000 Carcassonne, France

f.rey@laposte.net

Reçu le 5 mars 2001 Accepté le 22 octobre 2001

Fruits, 2002, vol. 57, p. 3-10 (C) 2002 Cirad/EDP Sciences All rights reserved DOI: 10.1051/fruits:2002001

Resumen Español, p. 10

\section{Silver rust bananas in Martinique: distribution and methods of control.}

Abstract - Introduction. Since 1996, silver rust damage, caused hitherto by Hercinothrips femoralis Reuter, has become very harmful for marketing. The present study aimed to collate information on the pest, its dispersion and the methods to control it. Materials and methods. The evolution of the populations and the damage of silver rust was followed in ten selected banana plantations for one year. Samples were taken on these plantations to check the identification of the thrips. To improve the control, standard bunch covers and insecticide- (cypermethrin or fipronil) impregnated bunch covers were tested. Results and discussion. The thrips Elixothrips brevisetis Bagnall seems to have replaced H. femoralis. It is today responsible for the damage of silver rust in Martinique and able to constantly pullulate throughout the year everywhere in the island. The standard bunch covers made it possible to protect $96 \%$ of the fingers, whereas, on bunches not covered, the parasitism rate was 98\%. Although the insecticide-impregnated bunch covers protect $100 \%$ of the fingers, their interest appears limited because of the constraints that they impose. Conclusion. To control the thrips, it is not the type of bunch cover which is the determining factor, but the date of the installation. A standard bunch cover, placed at the stage of the "last female hand discovered", appears to be the best method for protecting the bunches, and without using chemicals.

Martinique / Musa (bananas) / insect control / Elixotbrips brevisetis / rusts / damage / population distribution / control methods

\section{Rouille argentée des bananes martiniquaises : distribution et méthode de lutte.}

Résumé — Introduction. Depuis 1996, les dégâts de rouille argentée, occasionnés jusqu'alors par Hercinothrips femoralis Reuter, sont devenus très préjudiciables à la commercialisation. La présente étude a cherché à apporter quelques éléments sur le ravageur, sa dispersion et les méthodes de lutte. Matériel et méthodes. L'évolution des populations et des dégâts de rouille argentée a été suivie pendant un an, sur dix bananeraies sélectionnées. Des échantillons ont été prélevés sur ces plantations pour vérifier l'identification du thrips. Afin d'améliorer la lutte, des gaines standard et des gaines imprégnées d'insecticide (cyperméthrine ou fipronil) ont été testées. Résultats et discussion. Le thrips Elixothrips brevisetis Bagnall semble avoir remplacé $H$. femoralis. Il est aujourd'hui responsable des dégâts de rouille argentée en Martinique et capable de pulluler à tout moment de l'année sur l'ensemble de l'île. Les gaines standard ont permis de protéger $96 \%$ des doigts, alors que, sur régimes non gainés, le taux de parasitisme a été de $98 \%$. Bien que les gaines imprégnées d'insecticide protègent $100 \%$ des doigts, leur intérêt paraît limité en raison des contraintes qu'elles imposent. Conclusion. Dans la lutte contre ce ravageur, ce n'est pas le type de gaine mais la date de la pose qui est déterminante. Une gaine standard, posée au stade de la "dernière main femelle découverte ", paraît être le moyen le plus adapté pour protéger les régimes et, cela, sans utiliser d'intrant chimique.

Martinique / Musa (bananes) / lutte anti-insecte / Elixotbrips brevisetis / rouille / dégât / distribution des populations / méthode de lutte 


\section{Introduction}

Les dégâts de "rouille argentée " sur bananes sont dus à l'action de thrips qui, en se nourrissant, vident les cellules de leur contenu. Celles-ci, remplies d'air, sont à l'origine des plages décolorées et argentées visibles sur la peau des fruits. Elles brunissent et se subérifient au cours du temps, suite à l'oxydation des tanins [1]. Bien que superficielles, ces lésions sont préjudiciables à la commercialisation des fruits.

Les thrips mis en cause pour ces dégâts de rouille argentée sont Hercinothrips bicinctus (Bagnall) et Hercinothrips femoralis (Reuter) qui ont été signalés en Afrique du Sud [2], en Australie [3, 4], en Israël [5], à Hawaii et aux Canaries [4]. En Martinique, les premiers dégâts occasionnés par H. femoralis ont été observés en 1976, sur une plantation d'altitude $[6,7]$. Ce ravageur était alors considéré comme un parasite secondaire [1]. Cependant, les dégâts se sont nettement aggravés depuis 1996, avec des pertes à l'emballage pouvant atteindre $30 \%$. À cette même date, un autre thrips, Elixothrips brevisetis (Bagnall), a été identifié pour la première fois dans les zones de dégâts [8]. L'inquiétude grandissante des planteurs a conduit les groupements professionnels bananiers à demander aux structures de recherches du Cirad $^{1}$ d'actualiser les connaissances sur ce sujet.

Les travaux que nous avons menés ont cherché à faire le point sur la situation actuelle de la maladie et, en particulier, à identifier les thrips responsables de ces dégâts, à étudier leur distribution et à envisager des méthodes de lutte applicables.

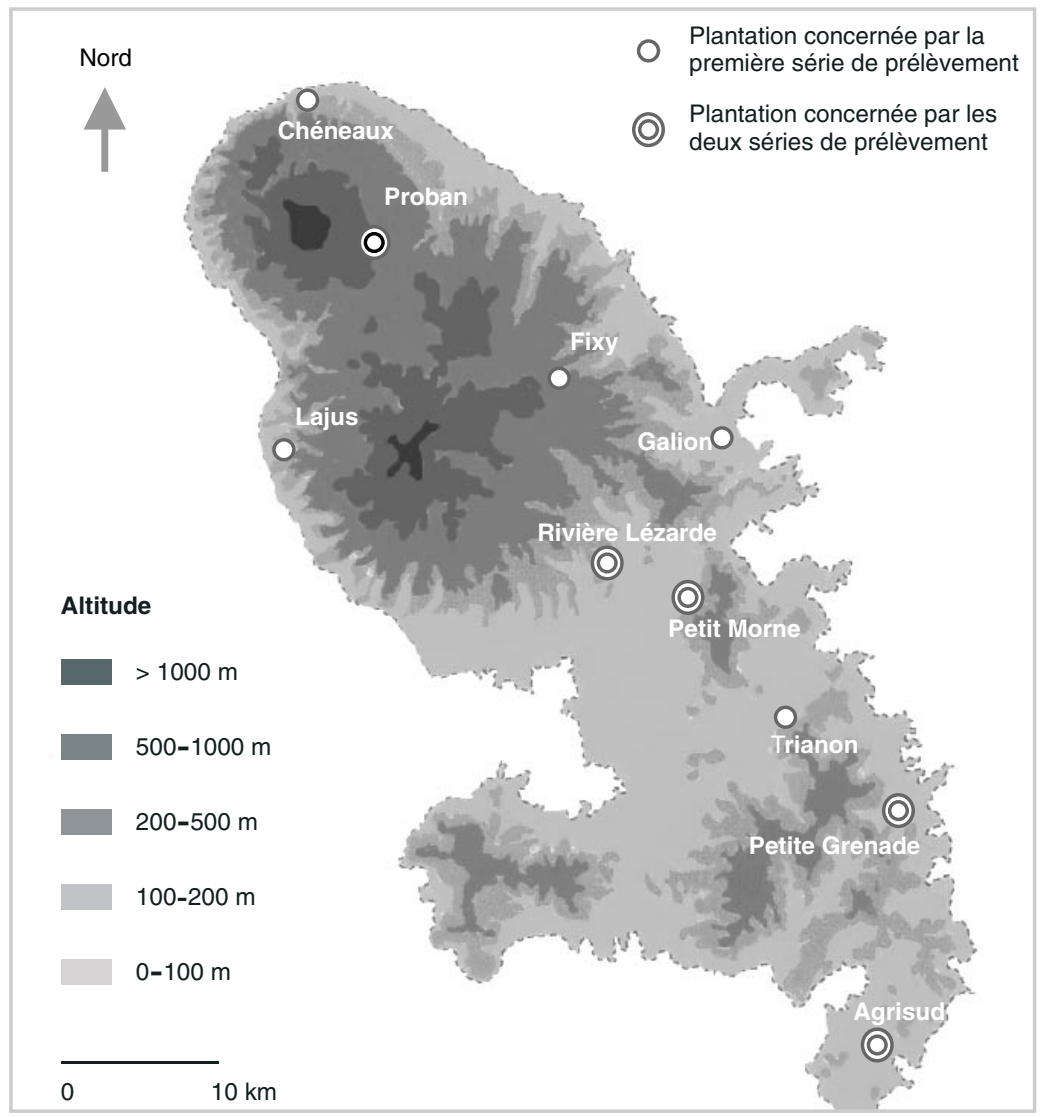

Figure 1.

Carte de la Martinique illustrant la localisation des bananeraies étudiées vis-à-vis des symptômes de « rouille argentée ».

\section{Matériel et méthodes}

\subsection{Prélèvement du ravageur pour identification}

Deux séries de prélèvements ont été réalisées à un an d'intervalle sur des plantations situées dans des zones climatiques et géographiques variées (figure 1). La première a été effectuée entre décembre 1999 et février 2000, sur les régimes de cinq plantations. La deuxième a été réalisée entre décembre 2000 et février 2001, sur les feuilles et régimes de dix plantations, dont les cinq précédentes.

Les insectes ont été récoltés à l'aide d'un pinceau, puis plongés dans de l'alcool éthylique à $10 \%$ additionné d'un mouillant (Teepol) à $1 \%$. Les thrips ont séjournés pendant 10 j dans cette solution, puis placés dans de l'alcool à $70 \%$. Les échantillons ont ensuite été identifiés ${ }^{2}$.

\subsection{Distribution du ravageur}

Un suivi d'un an a été mis en place en février 2000, sur différents secteurs de la Martinique, pour connaître l'évolution du niveau d'infestation sur des régimes non 
gainés et non traités avec des insecticides, ainsi que la distribution du ravageur sur quelques plantations ciblées, réparties sur l'ensemble de la Martinique.

Parmi quatre méthodes d'échantillonnage différentes (piégeage, berlèse, lavage des régimes et contrôle visuel) expérimentées, en 1975, par Delattre et Torregrossa [9], sur le thrips Chaetanaphothrips orchidii (Moulton) responsable de la rouille rouge sur banane en Guadeloupe et en Martinique, c'est la méthode du contrôle visuel qui s'était révélée la mieux adaptée. Sans pour autant prétendre accéder à une connaissance rigoureuse des oscillations de populations, les données recueillies par cette méthode, non destructive et ne perturbant pas le milieu, permettent une estimation rapide du niveau d'infestation des régimes.

Par ailleurs, en Australie, en 1994, pour un même type d'observations, Pinese et Piper [10] avaient effectué leurs comptages sur cinq régimes par hectare en évaluant de visu, par une note comprise entre 0 et 3 , le nombre de thrips adultes et le niveau de dégâts occasionnés par C. orchidii.

Le ravageur étudié étant du même type et les dégâts occasionnés étant assez comparables, la méthode que nous avons utilisée lors de notre étude a été dès lors basée sur une technique similaire.
Pour couvrir un maximum de situations, une dizaine de plantations ont été sélectionnées en fonction de critères pédologiques et climatiques diversifiés (figure 1): les sites de Chéneaux, Proban et Fixy sont situés en altitude où la pluviométrie est élevée (> $2500 \mathrm{~mm}$ ) ; les plantations de Galion, Rivière Lézarde et Petit Morne sont localisées dans une zone de collines et de plaines où la pluviométrie est moyenne (entre $2000 \mathrm{~mm}$ et $2500 \mathrm{~mm}$ ) ; les bananeraies de Lajus, Trianon, Petite Grenade et Agrisud se trouvent en bord de mer où le climat est plus sec $(<1500 \mathrm{~mm})$. Toutes les zones de production bananière de Martinique ont ainsi été représentées par au moins une plantation.

Sur chacune de ces plantations, une parcelle où les dégâts de rouille argentée étaient fréquemment observés a été choisie. L'intensité des dégâts et l'importance des populations de thrips présents sur cette parcelle ont été évaluées par une note visuelle attribuée à dix régimes (tableau I) ayant atteint un stade proche de la récolte, le grade du doigt médian de la main médiane étant compris entre (32 et 36) mm.

Tout au long de l'étude, le suivi a été effectué par le même observateur sur l'ensemble des parcelles.

Les parcelles de Petit Morne et d'Agrisud, adoptées comme parcelles de référence, n'ont subi ni traitement insecticide, ni gainage des régimes.

\section{Tableau I. \\ Échelle de notation des observations visuelles de la présence de thrips et de dégâts de « rouille argentée » sur bananiers.

$\begin{array}{ccc}\text { Note } & \text { Nombre de thrips } & \text { Dégâts observés } \\ 0 & 0 & \text { Absence de dégât } \\ 1 & \text { De } 1 \text { à } 10 & \begin{array}{c}\text { Quelques doigts attaqués } \\ \text { (au moins } 1 \text { doigt présente des dégâts) }\end{array} \\ 3 & \text { De } 11 \text { à } 20 & \begin{array}{c}\text { Quelques mains attaquées } \\ \text { (l'ensemble des doigts d'au moins une main présente des dégâts) }\end{array} \\ \text { Elus de } 20 & \text { Ensemble du régime attaqué } \\ \text { (tous les doigts du régime présentent des dégâts) }\end{array}$




\subsection{Test de l'efficacité de trois types de gaines}

À ce jour, aucun auxiliaire permettant la régulation des populations de thrips sur les cultures aux champs n'a été trouvé [11]. Par ailleurs, les traitements insecticides, outre les risques de pollution et de déstabilisation des écosystèmes qu'ils engendrent, posent de sérieux problèmes d'application. C'est pourquoi, pour contrôler les populations de ravageurs, nous avons préféré utiliser les techniques de contrôles physiques et de soins culturaux. Dans ce contexte, les gaines en polyéthylène placées autour des régimes sont couramment utilisées. Leur efficacité pourrait néanmoins être perfectionnée.

En Amérique centrale [12, 13] et en Australie [14], l'usage de gaines "prétraitées " avec un insecticide est très largement répandu ${ }^{3}$. Afin de déterminer l'efficacité de l'ensachage des régimes avec ces différents types de gaine vis-à-vis du thrips responsable de la rouille argentée, un essai a été mis en place en partenariat avec la société Addem plastiques. Il a été réalisé sur une parcelle dont les régimes n'avaient pas été traités avec des insecticides.

Trois types de gaines ont été testés : une gaine standard sans traitement, une gaine imprégnée de cyperméthrine ${ }^{4}$ et une gaine imprégnée de fipronil. Cet essai, effectué entre août et octobre 2000 sur une parcelle ne recevant pas d'insecticide (plantation de Petit Morne), a comporté quatre traitements : régime non gainé (t1) et régimes protégés par une gaine standard sans traitement $(\mathrm{t} 2)$ ou par une gaine imprégnée à la cyperméthrine à $0,21 \%$ de matière active (t3) ou par un gaine imprégnée au fipronil à 0,31 \% (t4).

Les gaines, fabriquées spécialement pour cet essai, ont été posées au stade " dernière main femelle découverte ". Un bananier correspondait à une répétition et chaque traitement a comporté 16 répétitions.

Un suivi de l'évolution du nombre de régimes présentant des dégâts (variable quantitative) a été effectué tous les 15 j sur l'ensemble des régimes non gainés du traitement t1. À la récolte, les doigts parasités de chacun des régimes ont été comptés, un " doigt parasité " correspondant à une banane montrant des dégâts de rouille argentée sur son épiderme. Les résultats ont été traités par une analyse de variance sur la variable " taux de doigts parasités".

\section{Résultats et discussion}

\subsection{Identification du ravageur}

En septembre 1996, E. brevisetis avait été identifié pour la première fois sur des zones de dégâts de rouille argentée, en Martinique [8]. H. femoralis y était jusqu'alors très répandu et décrit comme le thrips responsable de ce type de dégâts [1, 6, 15].

Parmi les 25 échantillons prélevés sur les feuilles et régimes de dix plantations martiniquaises, seuls des individus de l'espèce E. brevisetis ont pu être identifiés. L'espèce H. femoralis pourrait donc avoir disparu, remplacée par E. brevisetis. Cette évolution expliquerait l'intensification des pullulations observées depuis 1996.

Seules des femelles ayant été trouvées au cours de nos prélèvements, il semblerait que cette espèce se reproduise donc, en apparence, uniquement par parthénogenèse de type thélytoque, comme $C$. orchidii [2]. À Hawaii, depuis 1981, E. brevisetis occasionne sur banane le même type de dégâts que ceux observés en Martinique. Aucun mâle n'y a non plus jamais été observé [16].

Par ailleurs, Sakimura a rapporté, en 1985, que ce thrips, qu'il décrit comme un ravageur phytophage et polyphage, était présent dans le Pacifique et dans l'océan Indien [16]. En Martinique, larves et adultes de ce thrips ont été vus simultanément présents sur feuilles et sur régimes. Aucune étude sur les durées de développement de l'insecte n'a été publiée.

\subsection{Distribution du ravageur}

\subsubsection{Populations et dégâts sur régimes ni gainés, ni traités}

L'analyse de l'évolution des populations et des dégâts sur des régimes ni gainés, ni 
traités de deux plantations (Petit Morne et Agrisud) a montré que les populations du thrips E. brevisetis restaient à un niveau très élevé et relativement stable toute l'année (figure 2). Les notes moyennes attribuées aux régimes ont toujours été supérieures à 2, il y a donc eu constamment au moins 20 thrips par régime. Comme l'avaient observé Delattre et Torregrossa [7] pour C. orchidii, il n'y a pas eu d'arrêt du cycle évolutif et les colonies formées par les stades larvaires et adultes ont pu être observées à tout moment de l'année, avec recouvrement de différentes générations.

Les dégâts ont été très importants : les notes moyennes comprises entre 2 et 3 ont révélé que les doigts de plusieurs mains, voire de l'ensemble du régime, pouvaient être attaqués toute l'année.

Entre juin et août, une légère diminution de l'infestation a été notée sur la plantation de Petit Morne, alors que la pression parasitaire restait stable à Agrisud. Or, début juin à Petit Morne, la majorité des régimes âgés avaient été éliminés pour favoriser la repousse des rejets. Cette opération pourrait avoir induit une diminution de la population et une plus faible contamination des jeunes régimes restés en place. Les régimes non gainés proches de la récolte serviraient, par conséquent, de zone de refuge et de multiplication du ravageur. Sur les parcelles en production, il arrive que quelques régimes " oubliés " ne soient pas engainés. Ceux-ci susceptibles de jouer un rôle de réservoir devraient donc être supprimés.

\subsubsection{Infestation sur huit plantations martiniquaises}

À la différence des régimes ni gainés, ni traités, les fluctuations de populations sur les régimes des huit plantations traitées ont présenté de très grandes amplitudes (figure 3). E. brevisetis a été identifié sur chacune d'elles, qu'elles soient en altitude (zones humides) ou en bord de mer (zones plus sèches). Sur les plantations de Trianon, Galion, Fixy, Rivière Lézarde et Chéneaux, il a eu des pics de pullulation. En revanche, les bananeraies de Proban et Petite Grenade ont été peu touchées par ce thrips, et celle de Lajus a été constamment infestée avec 20 à 30 thrips en moyenne par régime,

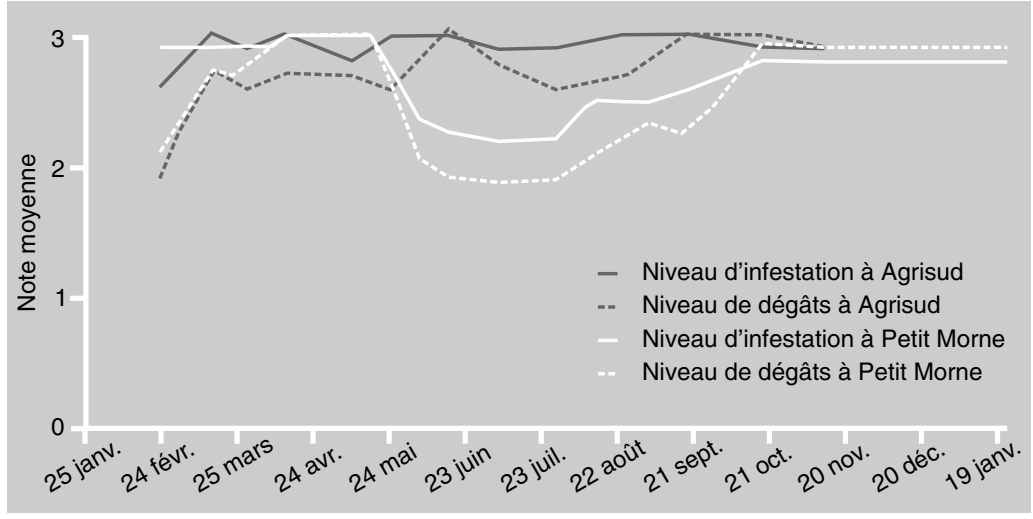

malgré la présence des gaines. Les pullulations ont pu être observées à tout moment de l'année.

La dynamique des populations de thrips sur une parcelle peut être influencée par de nombreux facteurs comme le climat, l'environnement et l'entretien.

La prise de nourriture et le taux de reproduction des thrips augmentent proportionnellement avec la température, jusque vers (30 à 35) ${ }^{\circ} \mathrm{C}$. L'humidité relative idéale à leur développement est comprise entre (70 et 90) \% [17]. Les conditions martiniquaises leur sont donc globalement favorables toute l'année. Cependant, des microclimats dans certaines parcelles peuvent accélérer leur développement.

E. brevisetis étant polyphage [16], il est probable que des plantes hôtes, autres que
Figure 2.

Évolution, au cours de l'année 2000, du niveau d'infestation et de l'intensité des dégâts bananeraies où les régimes n'ont été ni gainés, ni traités. La signification des notes de 0 à 3 est donnée dans le tableau I.

\section{Figure 3.}

Évolution, au cours de l'année 2000, du niveau d'infestation et de l'intensité des dégâts dus au thrips sur huit bananeraies martiniquaises où les régimes ont été des notes de 0 à 3 est donnée dans le tableau I. dus au thrips sur deux engainés. La signification

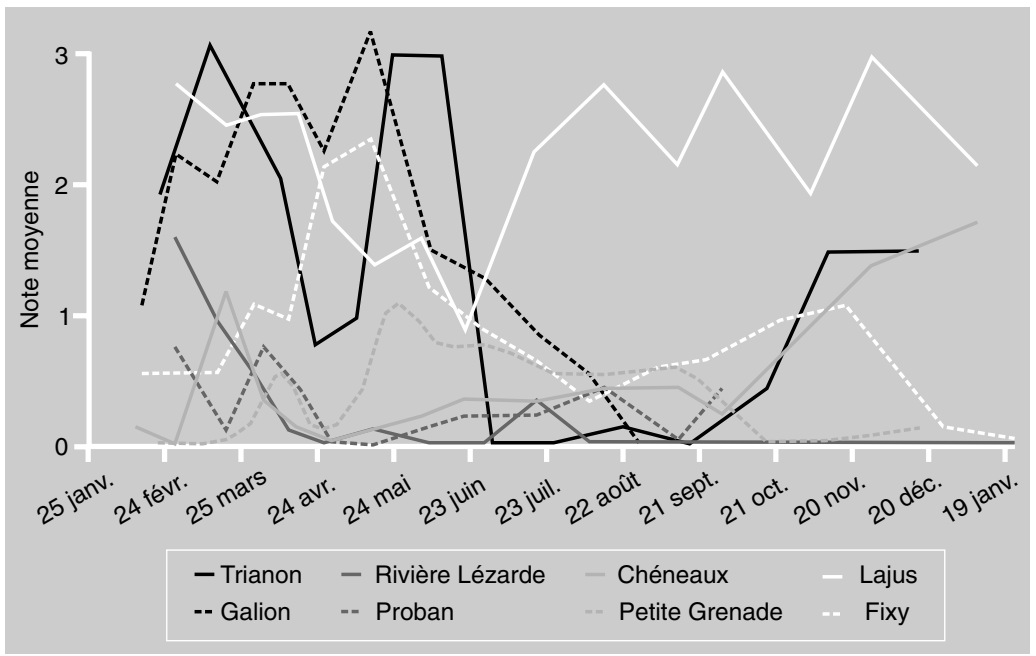


les bananiers, jouent un rôle de réservoir et entretiennent une pression parasitaire.

Les travaux d'entretien peuvent aussi avoir un impact sur les populations : lorsque les vieilles feuilles de bananiers sont coupées ou les rejets œilletonnés, les œufs et larves mis à terre ne peuvent pas poursuivre leur développement et meurent.

Cependant, comme nous l'avons constaté précédemment sur régimes ni gainés ni traités, les populations restent à un niveau relativement stable toute l'année. Il semble donc que les traitements insecticides et le retard à l'engainage soient les principaux facteurs responsables des fluctuations de population mises en évidences sur ces huit plantations traitées.

E. brevisetis serait donc présent toute l'année sur l'ensemble de la Martinique. Les causes des pullulations observées à certaines périodes et sur certaines exploitations restent difficiles à déterminer, bien qu'il semble que les retards dans les travaux d'entretien jouent un rôle déterminant.

À noter que l'évolution des dégâts a été similaire à celle des populations et qu'elle n'a donc pas apporté d'information supplémentaire.

\subsection{Efficacité comparée de trois types de gaines}

\subsection{1. Évolution du parasitisme sur régimes non gainés}

Au stade de la " dernière main femelle découverte " (dmfd), les régimes n'ont présenté encore aucun dégât (figure 4). En revanche, dès le quatorzième jour après ce

\section{Figure 4.}

Pourcentage moyen de " régimes non gainés présentant des dégâts » en fonction du nombre de jours écoulés à partir du stade de la " dernière main femelle découverte » (dmfd) observé au jour « 0 ». La récolte est faite $70 \mathrm{j}$ après le stade dmfd. stade, 19 \% des régimes ont montré des premiers symptômes. Cette rapide contamination s'est ensuite poursuivie puisque, 42 jours après le stade " dmfd ", tous les régimes non gainés étaient atteints.

Sur la plupart des plantations, la majorité du personnel est réquisitionné au hangar d'emballage au moment des pics de production, au détriment des travaux au champ. Il s'ensuit souvent un retard à l'engainage et la gaine est posée alors de (14 à 21) j après le stade " dmfd ". Or, les résultats de notre essai montre que, à ce moment là, (20 à 40) \% des régimes sont déjà contaminés (figure 4). Après engainage, les thrips en place vont pouvoir se développer et pulluler sous les gaines, à l'abri des changements brusques de température et dans une zone où l'humidité relative est élevée. Cela expliquerait en partie les fluctuations observées sur les plantations lors du suivi des huit bananeraies martiniquaises.

\subsubsection{Résultats des différentes modalités à la récolte}

La différence entre les nombres de doigts parasités sur régimes non gainés ( $\mathrm{t} 1=98 \%)$ ou gainés $(\mathrm{t} 2=4 \%, \mathrm{t} 3=0 \%$ et $\mathrm{t} 4=0 \%)$ a été trouvée hautement significative $\left(p=10^{-4}\right)$; l'efficacité du gainage serait donc évidente (tableau II).

Bien que la parcelle étudiée ait été très infestée puisque $98 \%$ des régimes non gainés présentaient des dégâts, les gaines standard sans traitement (t2) placées au stade " dmfd " ont permis d'obtenir $96 \%$ de doigts indemnes. Le gainage effectué au stade de la " dernière main femelle découverte " permet donc une bonne protection des régimes.

Les gaines imprégnées de cyperméthrine (t3) ou de fipronil (t4) ont permis une protection totale des régimes. Leur efficacité a été significativement supérieure $(p=0,03)$ à celle des gaines non traitées (t2). Néanmoins, le bénéfice qu'elles apportent est relativement faible par rapport aux contraintes qu'elles engendrent : risque de résidus dans les fruits, coût supérieur (gaine et pose) et augmentation des intrants chimiques sur la culture. Leur intérêt paraît donc limité. 
Tableau II.

Moyenne des pourcentages de doigts parasités par des thrips, en fonction de quatre traitements appliqués aux régimes de bananier afin de limiter la manifestation de « rouille argentée ».

\begin{tabular}{|c|c|c|}
\hline Modalité & $\begin{array}{c}\% \text { de doigts parasités } \\
\text { par régime }\end{array}$ & $\begin{array}{l}\text { Intervalle de confiance } \\
\qquad(\%)\end{array}$ \\
\hline Régime non gainé (t1) & 97,94 a & $\pm 1,60$ \\
\hline Régime protégé par une gaine standard (t2) & $4,06 \mathrm{~b}$ & $\pm 3,86$ \\
\hline $\begin{array}{l}\text { Régime protégé par une gaine } \\
\text { imprégnée de cyperméthrine (t3) }\end{array}$ & $0,44 \mathrm{c}$ & $\pm 0,54$ \\
\hline $\begin{array}{l}\text { Régime protégé par une gaine } \\
\text { imprégnée de fipronil ( } \mathrm{t} 4)\end{array}$ & $0,00 \mathrm{c}$ & $\pm 0,00$ \\
\hline
\end{tabular}

\section{Conclusion}

La substitution de Hercinothrips femoralis Reuter par Elixothrips brevisetis Bagnall depuis 1996 semble avoir aggravé les dégâts de rouille argentée en Martinique. Nos travaux ont montré que le risque de pullulation était présent toute l'année sur l'ensemble du département.

Les gaines standard sans imprégnation d'insecticide ont donné de très bons résultats, avec $96 \%$ de doigts indemnes, sous réserve qu'elles soient posées à temps, c'està-dire avant le stade de la " dernière main femelle découverte ". En cas de retard à l'engainage, le risque de pullulation est très élevé car la gaine ne peut plus alors jouer son rôle de protection, les thrips étant déjà présents lors de la pose. Les planteurs doivent donc veiller à engainer à temps.

Les gaines prétraitées avec un insecticide (cyperméthrine et fipronil) ont permis d'obtenir des fruits indemnes de dégâts, mais leur intérêt reste limité compte tenu des contraintes qu'elles imposent.

Les essais conduits lors de nos travaux ont montré qu'il était possible de protéger efficacement les régimes de bananes contre les attaques de thrips, sans avoir à recourir à des intrants chimiques.

\section{Remerciements}

L'auteur remercie M. Monnier (Addem Plastiques), partenaire du test de gaines et J.-P. Bournier (Cirad-Amis, Montpellier) qui s'est chargé de l'identification des thrips.

\section{Références}

[1] Simon S., Les acariens et les thrips sur bananier, Fruits 45 (num. spéc.) (1990) 72-76.

[2] Jones R.K., Control of banana silvering thrips and cotton mites on bananas, Citrus Subtrop. Fruit J. 534 (1978) 5-7.

[3] Treverrow N.L., Banana rust thrips and banana silvering thrips, Agfact H6.AE.2 (1st ed.), Wollongbar, Australia, 1987.

[4] Simmonds N.W., Bananas, Longman, Tropical Agriculture Series (2nd ed.), Edinburg, UK, 1966.

[5] Ben-Dov Y., Klein M., Weizman Z., Preliminary observations on the life history and control of the banana pest Hercinothrips femoralis (Reuter) (Thysanoptera: Thripidae) in Israël, Hassadeh 67 (2) (1986) 284-286.

[6] Lachenaud J.L., Hercinothrips femoralis, nouveau ravageur des bananeraies martiniquaises, Cirad, doc. interne, Martinique, 1978. 
[7] Delattre P., Torregrossa J.P., Abondance saisonnière, distribution et déplacement des populations du thrips de la rouille de la banane Chaetanaphothrips orchidii (Moulton) (Thysanoptera, Thripidae) aux Antilles françaises, Ann. Zool. Ecol. Anim. 10 (2) (1978) 149-169.

[8] Rosette C., Chabrier C., Bulletin spécial thrips du bananier, Avertissement SPV et FDGDEC, Martinique, 1996.

[9] Delattre P., Torregrossa J.P., Comparaison de quatre méthodes d'échantillonnage applicables à l'étude du thrips de la rouille de la banane Chaetanaphothrips orchidii (Thysanoptera, Thripidae), Nouv. Agron. Antill.Guyane 1 (3) (1975) 186-189.

[10] Pinese B., Piper R., Bananas insect and mite management, Lavoisier, Queensland, Australia, 1994.

[11] Parrella M.P., Lewis T., Integrated pest management (IPM) in field crops, in: Lewis T. (Ed.), Thrips as crop pests, CAB international, Wallingford, UK, 1997.
[12] Lara E.F., Problemas y procedimientos bananeros en la zona Atlántica de Costa Rica, Trejos Hnos, San José, Costa Rica, 1970.

[13] Henriques W., Feffers R.D., Lacher T.E., Kendall R.J., Agrochemical use on banana plantations in Latin America: perspectives on ecological risk, Environ. Toxycol. Chem. 16 (1) (1997) 91-99.

[14] Pinese B., Chlorpyriphos-impregnated bunch covers and insecticides control banana rust thrips, Qld. J. Agric. Anim. Sci. 44 (2) (1987) 113-116.

[15] Bournier A., Thysanoptères de Martinique et de Guadeloupe, Zoology (J. Pure Appl. Zool.) 3 (3) (1993) 227-240.

[16] Mau R.F.L., Martin Kessing J.L., Elixothrips brevisetis Bagnall, http://www.extento.hawaii. edu/kbase/crop/Type/e_brevis.htm, Dep. Entomol., Honolulu, Hawaii, 1993.

[17] Kirk W.D.J., Distribution, abundance and population dynamics, in: Lewis T. (Ed.), Thrips as crop pests, CAB international, Wallingford, UK, 1997.

\section{Roya plateada del banano en Martinica: distribución y métodos de control.}

Resumen - Introducción. Desde 1996, los daños de roya plateada, ocasionados hasta entonces por Hercinothrips femoralis Reuter, constituyen un gran perjuicio para la comercialización. Este estudio intentó aportar algunos elementos sobre la plaga, su dispersión y los métodos de control. Material y métodos. Se siguió, durante un año, la evolución de poblaciones y daños de roya plateada en diez plantaciones de banano seleccionadas. Se tomaron muestras en dichas plantaciones para verificar la identificación del trips. Con el fin de mejorar el control, se probaron bolsas estándar y bolsas impregnadas de insecticida (cipermetrina o fipronil). Resultados y discusión. El trips Elixothrips brevisetis Bagnall parece que haya reemplazado a $H$. femoralis. Actualmente, es el responsable de los daños de roya plateada en Martinica, siendo capaz de pulular en cualquier época del año por toda la isla. Las bolsas estándar permitieron proteger el 96\% de los dedos, mientras que, en racimos no embolsados, la tasa de parasitismo fue del 98\%. Aunque las bolsas impregnadas con insecticida protegen el 100\% de los dedos, su interés es limitado debido a las limitaciones que imponen. Conclusión. Para el control de esta plaga, no es tanto el tipo de bolsa sino la fecha de embolsado la que se muestra determinante. Una bolsa estándar, colocada en la fase de la "última mano femenina descubierta", parece ser el medio más adaptado para proteger los racimos y ello sin que se tengan que utilizar insumos químicos.

Martinica / Musa (bananas) / control de insectos / Elixotbrips brevisetis / roya / daños / distribución de la población / métodos de control

To access this journal online: www.edpsciences.org 